\title{
Note: Internal diamagnetic flux measurements on ASDEX Upgrade
}

\author{
L.Giannone, * R.Fischer, J.C.Fuchs, B.Geiger, M.Maraschek, D.Rittich, \\ B.Sieglin, A.Bock, J.Hobirk, A.Kallenbach, V.Mertens, and K.H.Schuhbeck \\ Max Planck Institute for Plasma Physics, 85748 Garching, Germany \\ P.J.McCarthy \\ Department of Physics, University College Cork, Cork T12 YN60, Ireland
}

\begin{abstract}
Internal diamagnetic flux measurements, with measurement loops and compensation magnetic probes inside the vacuum vessel, are now available on the ASDEX Upgrade tokamak. The measured diamagnetic flux is compared to that predicted by TRANSP simulations and calculated from equilibrium reconstruction. The diamagnetic flux measured at 2 positions separated toroidally by 180 degrees in the vacuum vessel are compared.

The diamagnetic flux is the small difference in total toroidal flux with plasma and without plasma [1]. A number of tokamaks have demonstrated the measurement of the diamagnetic flux in real-time using analog summation circuits [2-5]. The compensation for poloidal field and ohmic heating coil currents were carried out by analog subtraction of an appropriate fraction of the measured coil current. In superconducting tokamaks, where the toroidal field is constant before and after the discharge, it is possible to carry out a diamagnetic flux measurement without using an analog summation circuit [6-8]. The diamagnetic flux integrator can also be initiated in the flat top of the toroidal field current to make digital compensation of the signal possible [5, 9].

In earlier research work, the details and the theoretical background of external diamagnetic flux measurements were described [10]. It has a limited bandwidth of operation owing to the low pass filtering effect of the vacuum vessel. The improvement in bandwidth of diamagnetic flux measurements with internal measurement loops and compensation probes is the motivation for this work.

A schematic diagram of the internal and external diamagnetic flux measurements on ASDEX Upgrade is shown in Fig. 1. The internal measurement loop is a single winding in the poloidal direction around the inside of the vacuum vessel. This measures the toroidal magnetic flux generated by the toroidal field coils and the plasma. Magnetic probes oriented in the toroidal direction provide the internal compensation signal and measure the local toroidal flux generated by the toroidal field coils. These probes have a cross-section of $45 \mathrm{~mm} \times 68 \mathrm{~mm}$ and are located on the inner wall of the vacuum vessel. There are two sets of measurement loops and compensation probes separated by 180 degrees in the toroidal
\end{abstract}

\footnotetext{
* Louis.Giannone@ipp.mpg.de
}

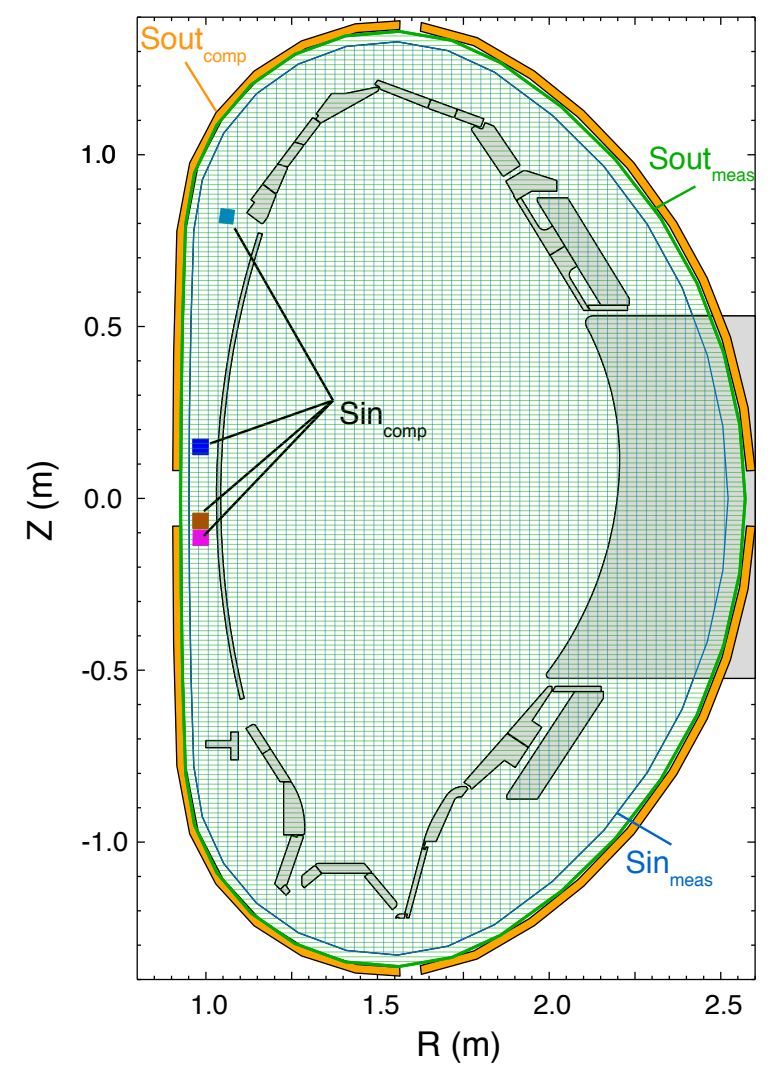

FIG. 1. Schematic diagram of the internal and external diamagnetic flux measurements on ASDEX Upgrade. The external measurement loop (green) is 2 turns mounted on a frame surrounding the vacuum vessel in the poloidal direction. The external compensation loop (yellow) is also mounted on this frame. The internal measurement loop (sea blue) is a single turn mounted on the inside vacuum vessel in the poloidal direction. Magnetic probes oriented in the toroidal direction provide the internal compensation signal.

direction. The diamagnetic flux is then simply the measurement loop flux minus the compensation probe flux.

Vacuum field measurements with a steady value of current in the toroidal field coil are used to establish the relative sensitivity of the measurement loop and compensation probe. The integrators of the measurement loop and compensation probe were triggered close to flat top phase of the toroidal field coil current. In the ideal case, the measurement loop and compensation probe of 


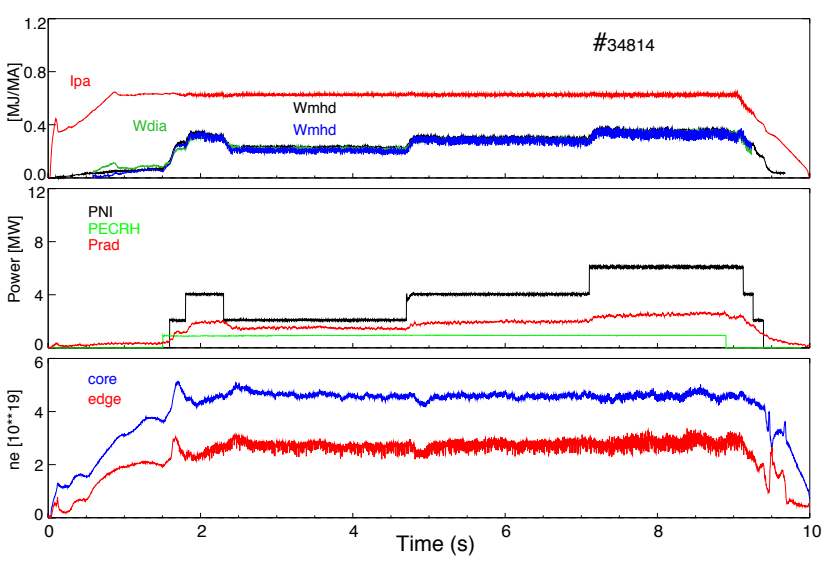

FIG. 2. Overview of discharge with constant ECRH and NBI power steps. The upper frame shows the time evolution of the plasma current (Ipa) and the plasma energy from the real-time (blue) and offline (black) equilibrium reconstruction (Wmhd) and inferred from the external diamagnetic flux (Wdia). The middle frame shows the time evolution of the neutral beam injection heating (PNI), electron cyclotron heating (PECRH) and radiated power (Prad). The lower frame shows the time evolution of the electron density line integral in the core and edge of the plasma.

the diamagnetic flux measurement are perfectly axisymmetric and are exactly perpendicular to the poloidal field and ohmic heating coils. Owing to a small misalignment, it is necessary to correct each signal for the contribution generated by their mutual inductance to these coils. The correction term proportional to the plasma current was determined by comparing two discharges with plasma parameters as identical as possible with negative and positive toroidal magnetic field.

The transport code, TRANSP [11], allows the diamagnetic flux (parameter DFLUX) to be calculated and compared to the experimental measurements . An offline equilibrium reconstruction code (IDE), with pressure constraints including the fast ion contribution, is also used to calculate the diamagnetic flux [12].

An overview of a discharge with constant electron cyclotron resonance heating $(\mathrm{ECRH})$ power and neutral beam injection heating (NBI) power steps is shown in Fig. 2. Internal measurement loop and compensation probe signals are shown in Fig. 3. It can be seen that plasma energy loss due to ELMs causes rapid changes in the compensation probe signal. In contrast, the measurement loop signal is not affected. This infers that toroidal flux conservation by the vacuum vessel plays a role. Flux conservation leads to the generation of a poloidal current in the vacuum vessel wall in response to the decrease in plasma beta after an ELM and the resulting increase in diamagnetic flux. The vacuum vessel wall currents generate the decrease in flux seen by the compensation probe. Shown in Fig. 4, is the good agreement of the internal and external diamagnetic flux measurements with the values predicted by TRANSP and the values calculated from the

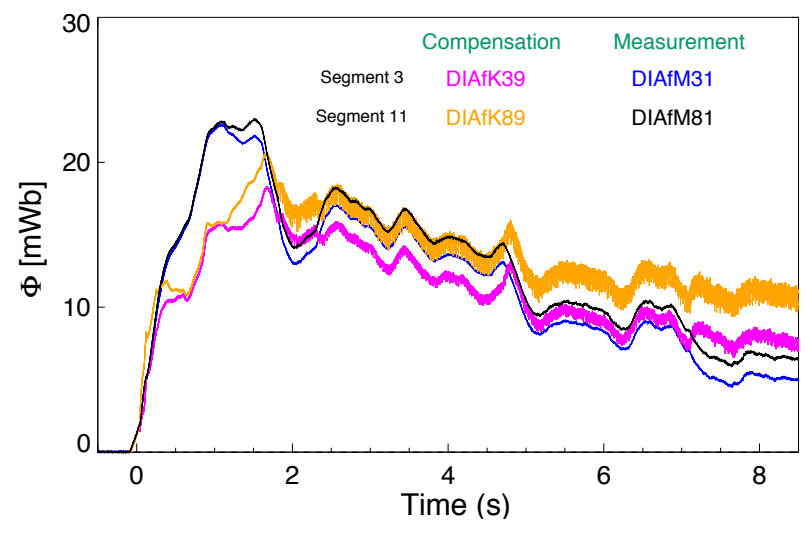

FIG. 3. Internal measurement loop and compensation probe signals for a discharge with constant ECRH power and NBI power steps. The measurement loops (DIAfM31,DIAfM81) and compensation probes (DIAfK39,DIAfK89) are at two locations separated toroidally by $180^{\circ}$. Plasma energy loss due to ELMs causes rapid changes in the compensation probe signal at times greater than $2 \mathrm{~s}$. In contrast, the measurement loop signal is not affected by ELMs.

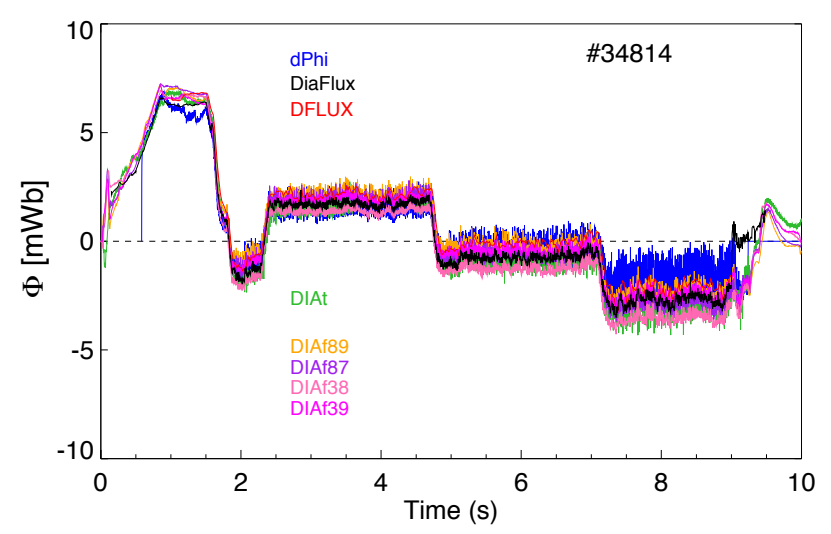

FIG. 4. Comparison of diamagnetic flux calculations and measurements. The predicted value from TRANSP (DFLUX), the calculated values from the real-time equilibrium reconstruction (dPhi) and offline equilibrium reconstruction (DiaFlux) and the external (DIAt) and 4 internal (DIAf89,DIAf87,DIAf38,DIAf39) diamagnetic flux measurements for the time interval of the discharge with auxiliary heating are in good agreement.

real-time and offline magnetic equilibrium reconstruction code in the NBI phase of the discharge.

The diamagnetic flux measurements in a small time window are shown in Fig. 5. The vacuum vessel has 16 segments. The internal diamagnetic flux measurements from segment 11 (DIAf89) show high frequency features between ELMs more strongly than in segment 3 (DIAf39). Closer inspection of the compensation probe signals shows that the higher frequency features are stronger in segment 11 than in segment 3 . The spikes in diamagnetic flux just after an ELM are also stronger in segment 11. The offline equilibrium reconstruction cal- 
culation of diamagnetic flux (DiaFlux) corresponds more closely to the diamagnetic flux measured in segment 3 . A comparison of poloidal magnetic probes at two toroidal positions (segment 5 and segment 13) shows that the precursor oscillations between ELMs are visible at both toroidal positions, with the magnetic probes on the inner vacuum vessel wall showing the largest signal.

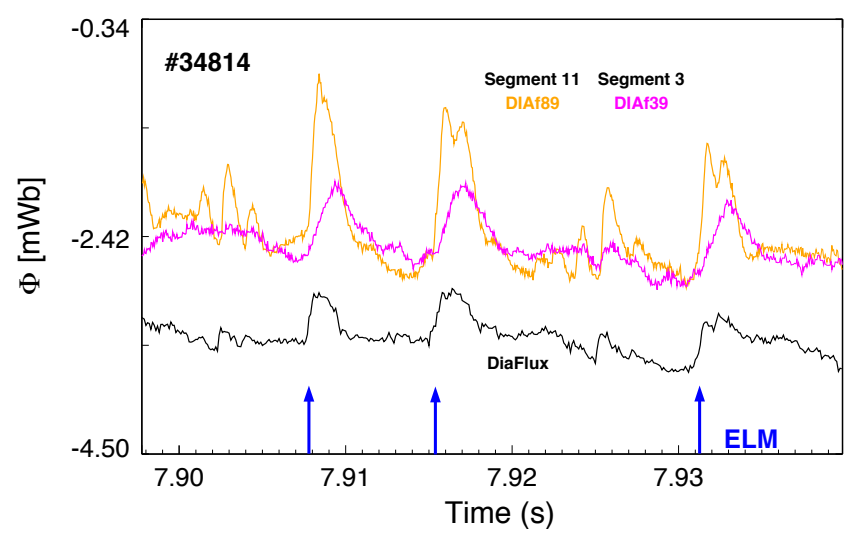

FIG. 5. Time evolution on a fast time scale of the measured and calculated diamagnetic flux. The internal diamagnetic flux measurements from segment 11 (DIAf89) shows high frequency features between ELMs more strongly than in segment 3 (DIAf39). The spikes in diamagnetic flux just after an ELM are also stronger in segment 11. The offline equilibrium reconstruction calculation of diamagnetic flux (DiaFlux) corresponds more closely to the measurements in segment 3.

Divertor tile currents are inferred from the voltage drop across shunt resistors mounted between the tiles and their mechanical support [13]. A comparison of divertor tile current measurements on inner and outer divertor tiles at three toroidal positions (segment 4, 12 and 14) shows that the precursor oscillations between ELMs are also visible at each toroidal position. The observed toroidal symmetry of the poloidal magnetic probe and divertor tile current signals makes the toroidal asymmetry of the internal diamagnetic flux difficult to explain, as it would require toroidal symmetry of the plasma at the separatrix and toroidal asymmetry of the plasma pressure collapse. It is also feasible that outward movement of the plasma to a region of smaller toroidal magnetic field or a change of plasma volume could produce changes in the diamagnetic flux [14]. Nevertheless, the observation of toroidal asymmetry of fast changes in diamagnetic flux indicates that caution is warranted when using these changes to calculate power losses to plasma facing components.

In summary, internal real-time diamagnetic flux measurements for ASDEX Upgrade are now in routine operation. The internal and external diamagnetic flux measurements, the predicted value by TRANSP and the calculated value from equilibrium reconstruction are found to be in good agreement.

In discharges with ELM's, the compensation probe signal responds on fast time scales similar to those seen in divertor tile current measurements. The measurement loop signal is not affected by the ELM. This is plausibly a consequence of toroidal flux conservation generating vacuum vessel currents. The vacuum vessel wall currents generate the decrease in flux seen by the compensation probe. The observed toroidal symmetry of the poloidal magnetic probe and divertor tile current signals in these discharges makes the toroidal asymmetry of the internal diamagnetic flux measurements difficult to explain.

The author would like to thank the integrator electronic development team (G.Sellmair, H.Eixenberger, L.Kammerloher, A.Wöls) for their vital contribution. The work leading to this article was funded by the European Atomic Energy Community and is subject to the provisions of the European Fusion Development Agreement. This work has been carried out within the framework of the EUROfusion Consortium and has received funding from the Euratom research and training programme 2014-2018 under grant agreement No 633053. The views and opinions expressed herein do not necessarily reflect those of the European Commission.
[1] J. Wesson, Tokamaks, 4th ed. (Oxford University Press, Oxford, England, 2011).

[2] J.Gernhardt and F. Schneider, Design and Electronic Compensation of a Diamagnetic Loop and its Applications in the ASDEX tokamak, IPP III-84 (Max-PlanckInsitut for Plasmaphysik, 1984).

[3] G. Tonetti, J. P. Christiansen, and L. de Kock, Rev. Sci. Instrum. 57, 2087 (1986).

[4] J. Moret, F. Buhlmann, and G. Tonetti, Rev. Sci. Instrum. 74, 4634 (2003).

[5] E. J. Strait, Rev. Sci. Instrum. 77 (2006).

[6] B. B. Shen, B. N. Wan, and X. Q. Zhang, Fusion Eng. Des. 70, 311 (2004).

[7] E. Joffrin and P. Defrasne, Rev. Sci. Instrum. 73, 2266 (2002).
[8] J. G. Bak, S. G. Lee, and H. S. Kim, Rev. Sci. Instrum. 82, 063504 (2011).

[9] H. Laqua and F. Schneider, Fusion Eng. Des. 48, 143 (2000).

[10] L. Giannone, B. Geiger, R. Bilato, et al., Rev. Sci. Instrum. 87, 0503509 (2016).

[11] J. Ongena, M. Evrard, and D. McCune, Fusion Science Technology 33, 371 (2004).

[12] R. Fischer, A. Bock, M. Dunne, et al., Fusion Sci. Technol. 69, 526 (2016).

[13] G. Pautasso, L. Giannone, O. Gruber, et al., Nucl. Fusion 51, 043010 (2011).

[14] V. Pustovitov, Plasma Phys. Control. Fusion 52, 085005 (2010). 